\title{
Author Index Vol. 11, 1996
}

Fetal Diagnosis Therapy

Abdellilah, M. 61 Abramovici, H. 341 Ahmad, F.K. 383 Aigrain,Y. 181 Anderson, E.M. 427

Antsaklis, A. 366 Arad,I. 271 Aravantinos, D. 366 Aubry,M.C. 199 Auslender, R. 341

Babay,Z.A. 221 Baldauf, J.-J. 57 Ballem,P. 210 Barahona, O. 146 Barbet,P. 154 Bar-Oz, B. 271

Bastert, G. 390 Becmeur, F. 57 Bellanti, J.A. 373 Bellotti,M. 106 Ben-David, Y. 341 Berrut, F. 57 Berry, S.M. 414 Bessho,T. 407 Bialer,M. 26 Bierkens, A.F. 137 Black, S.H. 402 Blanc, B. 357 Blanch, G. 32 Boemi, G. 132 Bondagji, N. 345 Boog,G. 72 Boubli,L. 357 Boudier, E. 37 Boute,O. 224 Brizot, M.d.L. 9 Bronshtein, M. 6 Bronstein, M. 301 Brookes, J.A.S. 417 Brosens, LA. 422 Bruno, M.T. 132 Buscaglia, M. 106 Butera, L. 132

Caione,P. 205 Calabrese, O. 94 Cantz, M. 114 Capitanucci, M.L. 205 Capozza, N. 205 Carreño, C.A. 348 Carroll, S.G. 1 Caruso, A. 264,313 Casanova, R. 191 Cha, S.C. 296 Chambers, K. 159 Chavinié, J. 154 Chen,C.-P. 150 Chervenak, F.A. 231 Cochrane, D.D. 159 Cowan, M.J. 241 Coyman, R.C. 146 Crang-Svalenius, E. 20 Cravello,L. 357 Crombleholme, T.M.

218 Cuckle,H.S. 254

Dadoun, K. 57 D’Alton, M.E. 218 Dansereau, J. 85 Dar,H. 99 Daskalakis, G. 366 David, A. 72

David, M. 99 Demarchi Aiello, V. 296 Deng, J. 417 Deprest, J.A. 422 Depret-Mosser, S. 224

D’Ercole,C. 357 De Santis, M. 264,313 Dilmen, G 50 Dommergues, M. 199 Dreyfus, M. 57

Drugan,A. 6, 16,301 Dudenhausen, J.W. 126 Dufour,Ph. 120 Dumez, Y. 199 Dungan,J.S. 398

Duquette, D.A. 414

Dvorin, E. 16 Dykes, A.-K. 20

Eddleman, K.A. 231 Ek,S. 318,326 Elliott, P.D. 221 Emerson, D.S. 398

Evans, A.A. 427 Evans, M.I. 6, 16,46, 254,301,306,348 Evrard, V.A.C. 422

Fahnenstich, H. 114 Farina, A. 94 Farquharson, D.F. 159, 210 Farriaux, J.P. 224 Favre, R. 335 Feitz,W.F.J. 137 Ferrazzi, E. 106 Ferro, F. 205 Flos,M.SJ. 137 Freedman, A. 46 Fugger, E.F. 402

Gardener, J.E. 417 Gasser, B. 79, 335 Gembruch, U. 286 George-Siegel, P. 231 Ghisoni, L. 106 Gilchrist, B.F. 67 Giovannini, G. 94 Golbus, M.S. 241 Conclaves, L. 46 Gross, B. 26 Guariglia, L. 260

Haddad,B. 61 Haddad,J. 37 Haentjens-Verbeke, K.

120 Hagglund, K.H. 383 Hahn,U. 390 Hajdu, K. 241 Hallak, M. 341

Hansmann, M. 114 Harman, C.R. 345 Harton,G.L. 402 Haverkamp, F. 114 Hawdon,J.M. 32 Haziza, J. 61 Hof,M. 286 Hofstaetter, C. 126 Huang, S.-H. 150 Hume, R.F., Jr. 6,46 Hwang, W.S. 221

Irvine, B. 159 Irwin,B. 159 Lsik, S. 50 Israel, N. 99 Itoh,S. 361 Itskovicz-Eldor, J. 6 Jackson, B.T. 67 Jacobs, D. 114 Jacques, S.M. 306,414 Jan,S.-W. 150 Jatene, A. 296 Johnson, J.-A.M. 85 Johnson, M.P. 6, 16,46,

301,306,383,414 Jones, D.R.E. 427 Jörgensen, C. 20 Jorizzo, G. 94 Junia Kajita, L. 296 
Kalousek, D.K. 85 Kaufman, G.E. 218 Khandelwal, M. 146 Kilpatrick, M.W. 402 King, M. 46 Kjeldgaard, A. 318,326 Komori, S. 407 Kornfeld,I. 210 Koyama, K. 407 Krivchenia, E.L. 16,254 Kubota, M. 275 Kurek,R. 390 Kuwabara, Y. 361

\section{KAÏUiEH}

E-Mail karger@karger.ch Fax+ 41613061234 http://www. karger. eh

(C) 1996 S. Karger AG, Basel

433

Labit,C. 357 La Ferrera, G. 132 Lais, A. 205 Lange, I.R. 221 Langer,B. 37,79, 191 Lanouette, J.M. 414 Lanzone, A. 132 LeBouc,Y. 154 Lees,W.R. 417 Lennert, T. 126 Lepercq, J. 154 Lerut,T.E. 422 Levinson, G. 402 Levi-Setti, P. 106 Lewin, F. 154 Littarru, GP. 264 Liu,D.T.Y. 427 Liu,F.-F. 150 Lopes, L.M. 296 Luks,F.I. 67 Luton, D. 154

McLean, L.K. 241 Mahieu-Caputo, D. 199 Mancuso, S. 132,313 Mandelbrot, L. 181 Manessier, L. 120 Manning, F.A. 345 Manouvrier, S. 224 Marconi, A.M. 106 Markling,L. 318,326 Martel,J. 345 Martin de Lassale, E.

224 Mastromarino, C. 264, 313 Mazumder, A. 373 Merksamer, R. 99 Michejda, M. 373 Migliaccio, A.R. 231 Migliaccio, G. 231 Millet, V. 357 Milunsky,A. 249 Mitsuhashi, N. 361 Monnier,J.C. 120,224

Montoya, Y. 191 Morichon-Delvallez, N.

199 Morrison, I. 345

Nakamura, Y. 361 Nakano, H. 275 Nasr,S. 254 Nebiolo,L. 249 Neumann, I. 126 Nicolaides, K.H. 1,9,

176 Nicolau,R. 79 Nijhuis,J.G 137 Noia,G 264,313

O’Brien, J.E. 16 Okamura,K. 169 Oradei,A. 264 Oury,J.F. 181 Ovadia,M. 292 Öztürk,M. 50 Pain,L. 37 Paniel,B.J. 61 Papageorgiou, I. 366 Pardi,G. 106 Pascal, O. 72 Patel,F. 9 Patricolo, M. 205 Pelizzola,D. 94 Petrikovsky, B. 26,292 Phillips, O.P. 398 Philpott-Howard, J. 1

Phylactou, L.A. 402 Piasecki, GJ. 67 Piffanelli, A. 94 Podevin, G. 181 Potier,A. 357 Pulvirenti, G 132

Quintero, R.A. 306,348 Qureshi,F. 306,414

Reece,E.A. 146 Reichler,A. 6,301 Resvani, E. 254 Ringdén,O. 318,326 Ritter, J. 57

Rodeck,C.H. 32,417 Romano, D. 313 Rosati, P. 260 Rotschild,A. 341

Sagot,P. 72 Sakata,K. 407 Satoh, S. 275 Sawai, H. 407 Schiesser, M. 390 Schlaeder,G. 37,79, 191 Schmitt,R. 146 Schneider, E. 292 Schulman, J.D. 402 Seifman,B. 306 Sherman, S.J. 383

Sheu,J.-C. 150 Shono,T. 275 Shulman,L.P. 398 Sibony,O. 154 Sideris, I. 176 Simeoni, U. 191 Singer, J. 85 Smith, C. 46,306 Snijders, R. 9 Sohn,C. 390 Sokol,RJ. 16,301 Sorokin, Y. 341 Spinazzola, R. 26 Steinbok, P. 159 Stoll,C. 79 Suita, S. 275

Tafas,T. 254 Taglioretti, A. 106 Takeuchi, H. 361 Talmant, C. 72 Tanaka, C. 361 Tanaka,H. 407 Tanigawara, S. 241 Tarabbia,C. 94 Tomlinson, M.W. 46 Toppare, M.F. 50 Tordjeman, N. 120 Touboul,C. 61 Trivellini, C. 264,313 Tsipouras, P. 402 Turhan,N.Ö. 50

Uzan, S. 61

Van Ballaer, P. 422 Vandenberghe, K. 422 Van Wymersch, D. 335 Velzen, D. van 32 Verma,U.N. 373 Vesce, F. 94 Ville,Y. 176 Vinatier,D. 120,224 Vollersen,E. 286 Vries, J.D.M. de 137 Vuillard,E. 181

Walkinshaw, S.A. 32 Wallwiener, D. 390 Weindling, A.M. 32 Weiner,C.P. 169 Wekemans, M. 199 Westgren,M. 318,326 Wildt, M.J.A.M. de 137 Wilson, R.D. 85, 159, 210 Winer, N. 72 Winsor, E.J.T. 85 Wittmann, B.K. 210 Wu,A.G. 373 
Yelian,F. 348 Yoshida,K. 361

Zamperini, P. 106 Zerres, K. 114

Zugaib,M. 296

434

Fetal Diagn Ther Vol. 11, 1996

Author Index 